\title{
Enhanced perioperative care and decreased cost and length of stay after elective major spinal surgery
}

\author{
Daniel A. Carr, DO, ${ }^{1}$ Rajiv Saigal, MD, PhD, ${ }^{1}$ Fangyi Zhang, MD, ${ }^{1,2}$ Richard J. Bransford, MD, ${ }^{2}$ \\ Carlo Bellabarba, MD, ${ }^{1,2}$ and Armagan Dagal, MD, FRCA, MHA ${ }^{2,3}$
}

Departments of ${ }^{1}$ Neurological Surgery, ${ }^{2}$ Orthopaedics and Sports Medicine, and ${ }^{3}$ Anesthesiology and Pain Medicine, Harborview Medical Center, University of Washington, Seattle, Washington

\begin{abstract}
OBJECTIVE The purpose of this study was to compare total cost and length of stay (LOS) between spine surgery patients enrolled in an enhanced perioperative care (EPOC) pathway and patients receiving traditional perioperative care (TRDC).

METHODS All spine surgery candidates were screened for inclusion in the EPOC pathway. This cohort was compared to a retrospective cohort of patients who received TRDC and a concurrent group of patients who met inclusion criteria but did not receive the EPOC (no pathway care [NOPC] group). Direct and indirect costs as well as hospital and intensive care LOSs were analyzed between the 3 groups.
\end{abstract}

RESULTS Total costs after pathway implementation decreased by $\$ 19,344$ in EPOC patients compared to a historical cohort of patients who received TRDC and $\$ 5889$ in a concurrent cohort of patients who did not receive EPOC (NOPC group). Hospital and intensive care LOS were significantly lower in EPOC patients compared to TRDC and NOPC patients.

CONCLUSIONS The implementation of a multimodal EPOC pathway decreased LOS and cost in major elective spine surgeries.

https://thejns.org/doi/abs/10.3171/2019.1.FOCUS18630

KEYWORDS ERAS; enhanced recovery after surgery; major spine surgery; perioperative medicine; cost analysis; length of stay; fast track; value

$\mathrm{E}$ NHANCED recovery after surgery (ERAS) initiatives attempt to standardize surgical care, reduce unnecessary variation, improve outcome, and reduce cost. This movement has taken hold in several surgical specialties including gynecology, ${ }^{13}$ hepatobiliary, ${ }^{11}$ urology, ${ }^{3}$ colorectal, ${ }^{6}$ head and neck, ${ }^{5}$ breast, ${ }^{22}$ and bariatric surgery. ${ }^{23}$ The ideas behind the ERAS guidelines of today were born from Dr. Kehlet's early work based on the attenuation of surgical stress, maintenance of postoperative physiological function, and early mobilization after surgery more than 2 decades ago. ${ }^{10}$

The application of ERAS to spine surgery is a next step for many institutions; however, it is an arduous undertaking requiring coordination of multidisciplinary perioperative service lines. Studies examining the benefits to patient outcome as well as cost reduction in spine and brain sur- gery are limited. Across many surgical specialties, there appears to be a cost-effectiveness to implementation of an ERAS pathway. ${ }^{21}$ In pediatric scoliosis surgery, accelerated discharge protocols focusing on preoperative patient education and multimodal analgesia have led to a decrease in hospital length of stay (HLOS) of 1.3 to 2 days and a $22 \%$ decrease in hospital charges. ${ }^{7,18}$ However, another study demonstrated that intraoperative variables such as implant and bone graft choices may influence cost more than an accelerated discharge program. ${ }^{17}$ Individual elements of ERAS such as intravenous acetaminophen have shown cost reduction in spine surgery. ${ }^{9}$ Standardization of preoperative medical assessment and optimization of patients allows preparation from a psychological and physical standpoint and may reduce inpatient LOS. Contemporary ERAS guidelines advocate for the implementation of ap-

ABBREVIATIONS CPT = Current Procedural Terminology; EPOC $=$ enhanced perioperative care; $E R A S=$ enhanced recovery after surgery; HLOS = hospital length of stay; ICD-9 = International Classification of Diseases, Ninth Revision; ILOS = intensive care length of stay; LOS = length of stay; NOPC = no pathway care; TRDC = traditional perioperative care.

SUBMITTED November 30, 2018. ACCEPTED January 21, 2019.

INCLUDE WHEN CITING DOI: 10.3171/2019.1.FOCUS18630. 
proximately 20 multimodal care elements in order for the treatment pathway to be successful. The marginal gains theory helps to explain this concept. Whereas individual care elements create only small unmeasurable changes, when they are aggregated as part of a multimodal pathway of care over time, they will lead to significant improvement in outcome.

Implementation of standardized care and reduction in unnecessary variation through ERAS requires significant institutional commitment. The process is best supported through education and data accrual to achieve the necessary culture change. Our institutional enhanced perioperative care (EPOC) program was developed to operationalize these objectives. ${ }^{4}$ The purpose of this study was to delineate the financial impacts of instituting an EPOC pathway for adult patients undergoing elective major spine surgery at a quaternary spine referral center.

\section{Methods}

Consecutive adult patients ( $>18$ years old) undergoing elective spine surgery were identified during preoperative assessment for inclusion in the EPOC pathway. This study was performed following the approval of the University of Washington IRB in Subcommittee ED; the requirement for written informed consent was waived by the IRB.

Patients were retrospectively reviewed as a historical cohort undergoing traditional perioperative care (TRDC) between $1 / 1 / 2012$ and 10/31/2013, and either the EPOC pathway or no pathway care (NOPC) between 12/1/2014 and 10/30/2017. The EPOC pathway database was created in 2013 and used for the analysis. Patients in the NOPC cohort met the same surgical inclusion criteria as the EPOC cohort but were not enrolled into the pathway due to administrative reasons such as scheduling conflicts, failure to attend preoperative clinic visits, and insurance authorization issues. The EPOC pathway was gradually introduced at Harborview Medical Center over a 13-month period from 11/1/2013 to $11 / 30 / 2014$. This transitional period was therefore excluded due to slow implementation of the full EPOC pathway and the variability in enrollment during this time. Patients in the TRDC group were identified by Current Procedural Terminology (CPT) and International Classification of Diseases, Ninth Revision (ICD-9) codes identified in the EPOC group including CPT 22800 , 22845, 22842, 22802, 22843, 22846, 22844, 22804, 22847, 22848, 63081, 63085, 63087, and 63090; and ICD-9 81.63, 81.64 , and 80.99. Briefly, these codes represent arthrodesis for spinal deformity; instrumentation codes for anterior > 2 levels or posterior $>3$ levels; corpectomy in the cervical, thoracic, or lumbar region; or pelvic fixation. All groups included patients treated by 4 spine surgeons who were practicing throughout the study periods.

Prior to implementation of the EPOC pathway, TRDC was performed in a noncoordinated fashion and variably by individual providers. Typically, in the TRDC group, patients were fasted at midnight and there was no consensus regarding perioperative multimodal analgesia. Early mobilization, early oral intake, and postoperative therapy were not coordinated and timely.

Application of the EPOC pathway for spine surgery patients was based on several surgical or medical criteria. Surgical inclusion criteria included anterior/posterior surgery, $\geq 4$-level nonrevision surgery, $\geq 3$-level revision surgery, revision anterior surgery, expected duration $>$ 6 hours, expected blood loss $>1$ L, planned staged surgery, corpectomy, or pedicle subtraction osteotomy. Medical criteria included poor functional status (arriving in a wheelchair, unable to complete activities of daily living or climb a flight of stairs), any daily home oxygen, use of continuous positive airway pressure/bilevel positive airway pressure (CPAP/BiPAP), unstable angina, implanted pacemaker, automatic implantable cardiac defibrillator or ventricular assist device, pulmonary hypertension, poorly controlled diabetes with hemoglobin Alc of $>8 \%$, hypertension or arrhythmias, and patients receiving anticoagulants, antiplatelets, or with coagulation disorders. Patients with current use of buprenorphine, naloxone, or Narcan, and those with intrathecal pain pumps or dorsal column stimulators were also included.

\section{Enhanced Perioperative Care}

The EPOC pathway is a multifaceted approach to preoperative, intraoperative, and postoperative care of the patient undergoing major spine surgery. Preoperative implementation focused on 5 aspects, including improvement of care coordination and patient education, carbohydrate loading preoperatively, preoperative multimodal analgesia, active warming prior to operating room, and nasal povidone-iodine swab application. Patient education preoperatively is important to set realistic goals and make sure the patient fully understands their operative intervention and postoperative course..$^{15,20}$ A 300-ml clear carbohydrate load was given 2 hours prior to hospital arrival and the night before to help attenuate the surgical stress and insulin resistance by providing perioperative nutrition. Multimodal analgesia was started in the preoperative area in the form of drugs with different mechanisms of action, including $1 \mathrm{~g}$ of acetaminophen the night before and on the morning of surgery as well as $1.2 \mathrm{~g}$ of gabapentin given in preoperative area.

Intraoperatively, patient temperature was maintained by continued active warming with intravenous fluid warmers, and keeping the patient covered and room temperature elevated prior to draping. Total intravenous anesthesia with propofol and remifentanil was used to maintain anesthesia. Four milligrams of intravenous ondansetron was administered for nausea and vomiting prophylaxis. For intraoperative analgesia ketamine was infused at $0.5 \mathrm{mg}$ / $\mathrm{kg}$ bolus and $0.5 \mathrm{mg} / \mathrm{kg} / \mathrm{hr}$ (based on ideal body weight) throughout the case, and intravenous acetaminophen was redosed at 6 hours intraoperatively. ${ }^{12,14}$ Fluid management was performed under goal direction by using pulse pressure variability, stroke volume variability, or cardiac output. ${ }^{2}$ Tranexamic acid $(1 \mathrm{~g})$ was given as a bolus prior to incision, and $1 \mathrm{~g}$ was given over 8 hours when massive blood loss was expected and under the direction of the attending surgeon.

Postoperatively, oral intake was encouraged in the postanesthesia care unit and resumption of full diet on postoperative day 1. Maintenance intravenous fluids were limited to $2 \mathrm{ml} / \mathrm{kg} / \mathrm{hr}$ to achieve zero balance. The Foley catheter 
TABLE 1. Demographic information for patients who underwent spine surgery with or without EPOC

\begin{tabular}{|c|c|c|c|c|c|}
\hline Variable & TRDC, $n=183$ & EPOC, $n=620$ & NOPC, $n=129$ & p Value, EPOC vs TRDC & $p$ Value, EPOC vs NOPC \\
\hline Age in yrs, mean \pm SD & $61 \pm 14$ & $60 \pm 13$ & $58 \pm 13$ & $0.3^{*}$ & $0.1^{*}$ \\
\hline Male (\%) & $80(44)$ & $298(48)$ & $57(44)$ & $0.3 \dagger$ & $0.4 \dagger$ \\
\hline $\mathrm{BMI}>30(\%)$ & $68(37)$ & $260(42)$ & $52(40)$ & $0.2 \dagger$ & $0.7 \dagger$ \\
\hline ASA score (\%) & & & & $0.07 \ddagger$ & $0.06 \ddagger$ \\
\hline I & $1(0)$ & $0(0)$ & $2(2)$ & & \\
\hline II & $80(44)$ & $248(40)$ & $56(43)$ & & \\
\hline III & $97(53)$ & $326(53)$ & $67(52)$ & & \\
\hline IV & $5(3)$ & $46(7)$ & $4(3)$ & & \\
\hline $\mathrm{CMI}$, mean $\pm \mathrm{SD}$ & $6.1 \pm 3$ & $5.5 \pm 2.5$ & $5.7 \pm 2.2$ & $<0.01^{*}$ & $0.3^{*}$ \\
\hline $\mathrm{CCl}(\%)$ & & & & $0.6 \ddagger$ & $<0.01 \ddagger$ \\
\hline 0 & $16(9)$ & $34(5)$ & $77(60)$ & & \\
\hline 1 & $110(60)$ & $382(62)$ & $25(19)$ & & \\
\hline 2 & $38(21)$ & $150(24)$ & $17(13)$ & & \\
\hline$\geq 3$ & $19(10)$ & $54(9)$ & $10(8)$ & & \\
\hline Payor mix (\%) & & & & $0.5 \ddagger$ & $0.7 \ddagger$ \\
\hline Commercial & $35(19)$ & $134(22)$ & $25(19)$ & & \\
\hline Exchange & $0(0)$ & $5(1)$ & $0(0)$ & & \\
\hline Medicaid & $33(18)$ & $106(17)$ & $29(22)$ & & \\
\hline Medicare & $95(52)$ & $307(50)$ & $59(46)$ & & \\
\hline Self-pay & $1(1)$ & $1(0)$ & $0(0)$ & & \\
\hline Other & $19(10)$ & $67(11)$ & $16(12)$ & & \\
\hline OR time in mins, mean $\pm \mathrm{SD}$ & $515 \pm 142$ & $439 \pm 164$ & $436 \pm 139$ & $<0.01^{*}$ & $0.5^{*}$ \\
\hline \multicolumn{6}{|l|}{ Service line $(\%)$} \\
\hline Orthopedic & $121(66)$ & $448(72)$ & $71(55)$ & & \\
\hline Neurological & $62(34)$ & $172(28)$ & $58(45)$ & & \\
\hline
\end{tabular}

$\mathrm{ASA}=$ American Society of Anesthesiologists; $\mathrm{BMI}=$ body mass index $\mathrm{CCl}=$ Charlson Comorbidity Index; $\mathrm{CMI}=$ case-mix index; $\mathrm{OR}=$ operating room.

* Student t-test.

$\dagger$ Chi-square test.

$\ddagger$ Chi-square test for trend, Fisher's exact test.

was removed by postoperative day 2 . The patient was required to sit up out of bed by postoperative day 2 . Ketamine was continued at $8 \mathrm{mg} / \mathrm{hr}$ for the first 24 hours after surgery, and $1 \mathrm{~g}$ of acetaminophen and $900 \mathrm{mg}$ of gabapentin were given for 3 days postoperatively.

\section{Cost Analysis}

Direct costs were broken into cost A and cost B to evaluate items that may have been impacted by EPOC pathway implementation. The universal billing grouping for the revenue code was used to group like services within a category predefined by the Centers for Medicare and Medicaid Services for the Universal Billing Form (UB04). Revenue codes were assigned to each billable service item for each unique department providing patient care services. The accounting "matching principle" was followed to ensure that expense and revenue were matched to the fiscal period. Direct costs A included room charges, pharmacy, emergency services, imaging, implants, laboratory, pathology, perioperative services, transfusions, and supply services. Direct costs B included implant and supply costs that should not have been affected by implementation of the perioperative clinical care pathway, other than reflecting improvement in purchasing contracts. Indirect costs included both shared patient service resources (such as patient transport, environmental services, medical equipment maintenance, resident salaries, and interpreter services) and nonpatient areas (such as building depreciation, medical records, hospital administration, and human resources). Whereas indirect costs cannot be matched to a specific patient, direct costs within patient care areas are under the control of the unit manager/director and can be linked to a patient by the service items used within that patient care area.

TRDC costs were adjusted for inflation from 2012 to 2017 by using the consumer price index from Seattle (http:// www.seattle.gov/financedepartment/cpi/documents/ Seattle_CPI_History_--_Annual_001.pdf).

\section{Statistical Analysis}

Analysis was performed using Statistical Package for the Social Sciences version 21 (SPSS). The mean LOSs were compared using the Student t-test with equal variances not assumed. Demographic comparisons were made using the chi-square test and Student t-test as indicated in Table 1. Financial costs were compared using the 2-tailed t-test. 


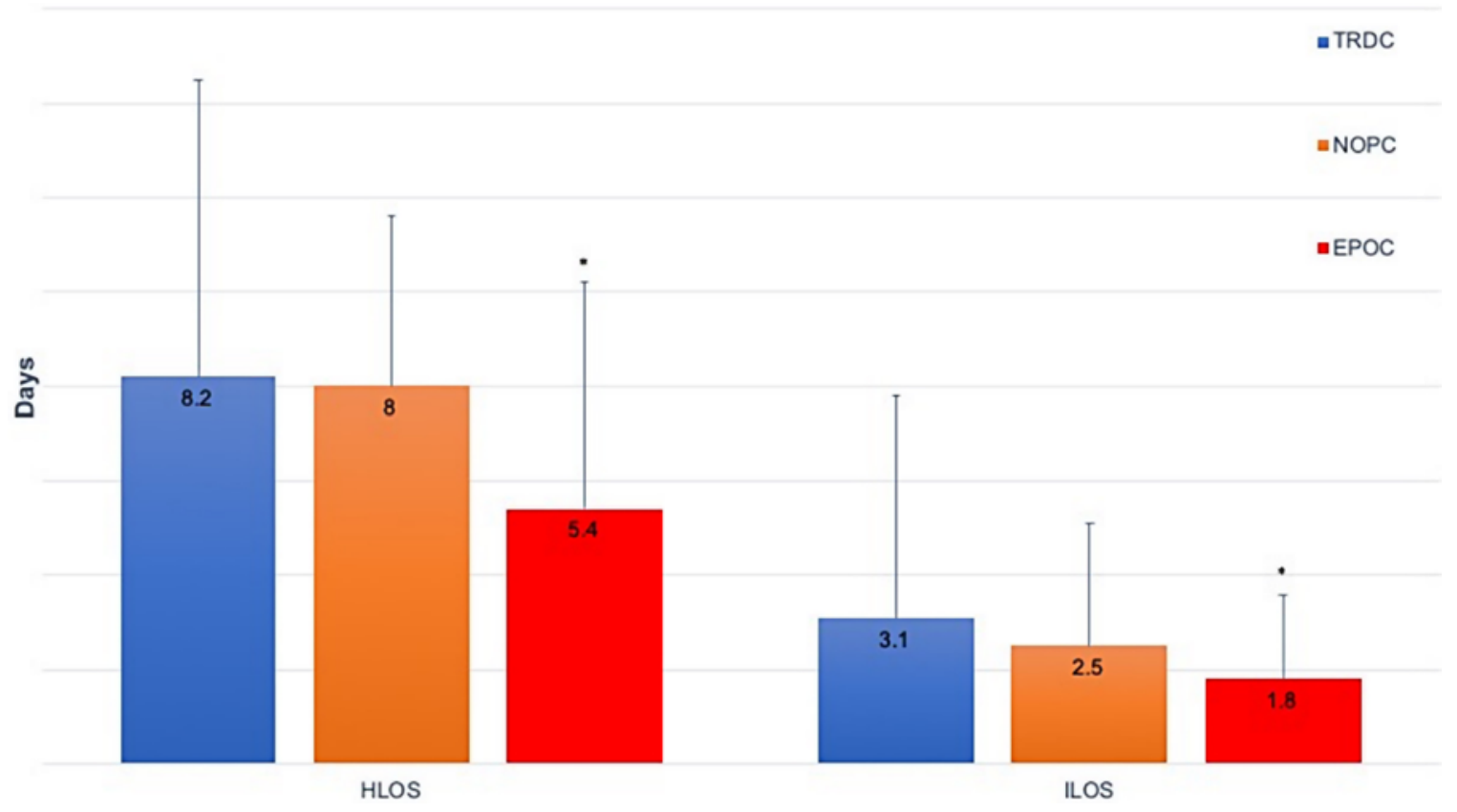

FIG. 1. Bar graph showing that EPOC significantly reduces HLOS and ILOS. Error bars represent the standard deviation. Asterisks represent statistical significance for TRDC versus EPOC $(p<0.01)$ and NOPC versus EPOC $(p<0.02)$. Student t-test, equal variance not assumed.

\section{Results}

Six hundred twenty patients were enrolled into the EPOC pathway. One hundred eighty-three TRDC patients and 129 NOPC patients were compared to the EPOC pathway patients. Patient demographic data are listed in Table 1. Of note, patients throughout all groups were similar in age, sex, body mass index, American Society of Anesthesiologists score, Charlson Comorbidity Index, and insurance type. The hospital case-mix index, a measure of patient complexity based on diagnosis-related group codes, was similar between NOPC and EPOC but was significantly higher in the TRDC group. Duration of operation was longer in the TRDC group than in the EPOC group, but was similar between EPOC and NOPC.

EPOC pathway patients had a significantly lower HLOS and intensive care LOS (ILOS) when compared to both TRDC and NOPC as outlined in Fig. 1. The HLOS was on average 8.2 days for TRDC, 8.0 days for NOPC, and 5.4 days for EPOC cohorts. The ILOS was on average 3.1 days for TRDC, 2.5 days for NOPC, and 1.8 days for EPOC patients. EPOC patients' LOS at both the hospital and intensive care levels was significantly lower than that for patients in the TRDC and NOPC groups.

Total cost was significantly lower in the EPOC patients than in TRDC (by 29\%) and NOPC (by 11\%). Overall cost savings of EPOC pathway per-patient care were $\$ 19,344$ compared to TRDC and $\$ 5889$ compared to NOPC. There was no significant difference in implant and supply costs (direct costs B) between NOPC and EPOC, but there was when comparing TRDC to EPOC, as noted in Table 2. For other direct costs (direct costs A), a cost savings of $\$ 11,160$ was identified in EPOC patients compared to TRDC and a $\$ 4102$ cost savings was identified in EPOC patients compared to NOPC. Direct costs A were significantly lower

TABLE 2. Financial outcomes in patients who underwent spine surgery with or without EPOC

\begin{tabular}{|c|c|c|c|c|c|c|c|c|}
\hline Cost (US\$) & $\begin{array}{l}\text { Mean Difference } \\
\text { (TRDC vs EPOC) }\end{array}$ & $\begin{array}{l}\text { Lower Cl } \\
(95 \%)\end{array}$ & $\begin{array}{l}\text { Upper Cl } \\
(95 \%)\end{array}$ & $\begin{array}{c}p \\
\text { Value* }^{*}\end{array}$ & $\begin{array}{l}\text { Mean Difference } \\
\text { (NOPC vs EPOC) }\end{array}$ & $\begin{array}{l}\text { Lower Cl } \\
(95 \%)\end{array}$ & $\begin{array}{l}\text { Upper Cl } \\
(95 \%)\end{array}$ & $\begin{array}{c}p \\
\text { Value* }^{*}\end{array}$ \\
\hline Direct costs A† & $\$ 11,160$ & $\$ 8,266$ & $\$ 14,054$ & $<0.01$ & $\$ 4,102$ & $\$ 2,201$ & $\$ 6,002$ & $<0.01$ \\
\hline Direct costs B $\ddagger$ & $\$ 2,837$ & $\$ 1,350$ & $\$ 4,323$ & $<0.01$ & $-\$ 1,052$ & $-\$ 2,543$ & $\$ 438$ & 0.2 \\
\hline Indirect cost & $\$ 5,140$ & $\$ 3,085$ & $\$ 7,195$ & $<0.01$ & $\$ 2,839$ & $\$ 1,400$ & $\$ 4,280$ & $<0.01$ \\
\hline Total cost & $\$ 19,344$ & $\$ 13,579$ & $\$ 25,109$ & $<0.01$ & $\$ 5,889$ & $\$ 1,766$ & $\$ 10,012$ & $<0.01$ \\
\hline
\end{tabular}

EPOC significantly reduces direct costs A, indirect costs, and total cost versus TRDC and NOPC. TRDC cost inflation was adjusted from 2013 to 2016. Cl (95\%) expressed as $\mathrm{Cls}$ for the difference, equal variances not assumed.

* 2-tailed t-test.

$\dagger$ Includes direct cost items that are likely to be affected by the clinical care improvement (pharmacy, bed and nursing, clinical service, emergency service, imaging, laboratory medicine, perioperative service, procedure and tests, transfusion and related blood services, rehabilitation, or other).

$\ddagger$ Includes direct cost items that are not likely to be affected by the clinical care improvement (implant and supply cost). 


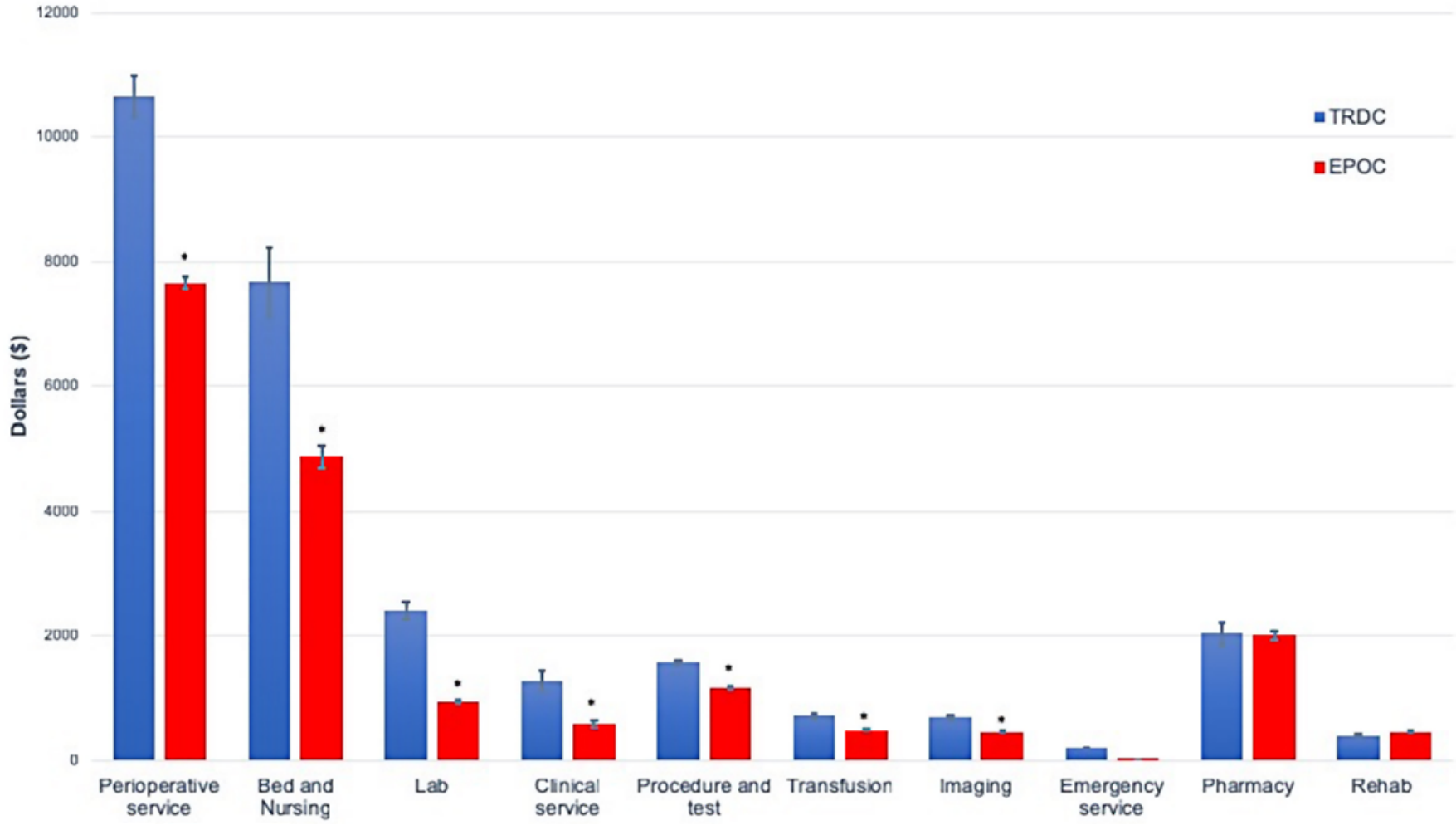

FIG. 2. Bar graph showing that EPOC reduces subcategories of direct costs versus TRDC. Error bars represent the standard error of the mean. Asterisks represent statistical significance for TRDC versus EPOC; values listed in Table 3. 2-tailed t-test.

for EPOC patients compared to TRDC in perioperative services, bed and nursing, laboratory, clinical services, procedures, transfusion, and imaging, as noted in Fig. 2 and Table 3. Costs of pharmacy services and rehabilitation services were similar between the TRDC and EPOC patients. Indirect costs were also lower in the EPOC patients compared to both TRDC and NOPC patients, as noted in Table 2.

\section{Discussion}

Enhanced recovery after spine surgery is still in its in- fancy, having been evaluated in only a limited number of studies to date. ${ }^{1,8,24,25}$ The ability to modify patient experience and outcome based on a multimodality approach is the cornerstone of the ERAS philosophy. Some reports of ERAS in spine have focused on perioperative strategies, while Wang et al. chose to develop their initial ERAS pathway around minimally invasive surgery to reduce surgical stress. ${ }^{25}$ However, most spine procedures are performed in an open, traditional fashion. We attempted to improve on major elective spine surgery through the perioperative strategies of ERAS. Included in these major spine surger-

TABLE 3. Direct cost A contributors in patients who underwent spine surgery with or without EPOC

\begin{tabular}{lcccc}
\hline \multicolumn{1}{c}{ Direct Cost A (US\$) } & Mean Difference (TRDC vs EPOC) & Lower Cl (95\%) & Upper Cl (95\%) & $p$ Value* \\
\hline Periop services & $\$ 2,996$ & $\$ 2,289$ & $\$ 3,702$ & $<0.01$ \\
\hline Bed and nursing & $\$ 2,793$ & $\$ 1,618$ & $\$ 3,968$ & $<0.01$ \\
\hline Lab medicine & $\$ 1,471$ & $\$ 1,200$ & $\$ 1,742$ & $<0.01$ \\
\hline Clinical services & $\$ 692$ & $\$ 224$ & $\$ 1,160$ & $<0.01$ \\
\hline Procedure and tests & $\$ 407$ & $\$ 289$ & $\$ 525$ & 0.04 \\
\hline Transfusion services & $\$ 238$ & $\$ 100$ & $\$ 375$ & $<0.01$ \\
\hline Imaging & $\$ 41$ & NA & $\$ 309$ & $<0.01$ \\
\hline Emergency services & $\mathrm{NA}$ & $-\$ 340$ & $\$$ & $\mathrm{NA}$ \\
\hline Pharmacy services & $\$ 34$ & $-\$ 110$ & $\$ 140$ & 0.86 \\
\hline Rehabilitation services & $-\$ 48$ & & 0.13 \\
\hline
\end{tabular}

$N A=$ not applicable; there was no emergency service use in the EPOC period.

EPOC reduces various subcategories of direct costs versus TRDC. Cl (95\%) expressed as Cls for the difference, equal variances not assumed. * 2-tailed t-test. 
ies are a recent rising rate of multilevel fusions in patients of all ages from 60 to 80 years old. ${ }^{19}$

The average surgical cost for multilevel fusions can exceed $\$ 100,000$, which represents an opportunity to save through faster discharge and decrease in complications. ${ }^{16,19}$ The direct costs related to supply and implants were significantly different between TRDC and EPOC groups. Among the NOPC and EPOC pathway cohorts, which represented the same time frame, the direct costs related to implants and supply were not different. However, TRDC implant and supply costs were higher than in the NOPC and EPOC patients and may therefore have been influenced by variables such as a change in surgical strategy or in hospital supply costs over time.

When breaking down the direct costs $\mathrm{A}$, which were expected to change, there was a difference between EPOC and TRDC pathways. Bed and nursing costs, although fixed, may have declined during the EPOC period due to shorter inpatient stays. Also, in accordance with better fluid management, selective use of tranexamic acid, and better preoperative comorbid disease optimization, the need for and therefore the cost of postoperative imaging, laboratory testing, and transfusions was noted to decrease. These patients also spent less time in the ICU, probably because of lower postoperative fluid overload, early enteral nutrition, and early mobilization postoperatively. We have not observed changes in pharmacy direct cost, probably because of the number of perioperative analgesics used, drug availability, and cost fluctuations in the pharmaceutical industry, including the use of intravenous acetaminophen. The cost of perioperative services declined after EPOC implementation, probably because of decreased postanesthesia care unit times. However, this metric was not available to evaluate in more detail at the time of submission.

Patients who were in the NOPC group did not get the opportunity for detailed preoperative assessment, optimization, education, discharge planning, and care organization. However, the care elements such as intraoperative, postoperative ICU, and ward care were standardized during the EPOC and NOPC time frame. This probably contributed to a reduction in overall costs between EPOC and NOPC. The EPOC pathway in major spine surgery achieved similar results to 2 previously reported "accelerated discharge" pathways in adolescent idiopathic scoliosis, including decreased cost and decreased LOS.,18 These results are in contrast to those of a recent publication about ERAS for metastatic spine lesions, which found no benefit to LOS but did find a decrease in postoperative pain scores. ${ }^{8}$

Our study has several limitations. The major limitation is the retrospective nature of the TRDC group. While demographically similar to EPOC and NOPC patients, the time frame of surgical care was different in the TRDC group and may be biased by surgeon experience, anesthesia staff differences, and hospital policy differences. However, all surgeons included remained the same during the historical cohort and the EPOC time frame. Case-mix index differences may be accounted for by the difference in time of the historical TRDC cohort and the more recent NOPC and EPOC cohorts. Also, the TRDC patients were identified using only CPT billing codes for major spine surgeries, whereas the EPOC pathway group also includes patients who had significant medical comorbidities.

We attempted to mitigate the limitation of this retrospective comparison by adding the NOPC group. Further details of the specific reason why each NOPC patient was not entered into the EPOC pathway are unknown. There may be confounding factors for these patients for which we cannot control. However, even patients in the NOPC group may have benefitted from some aspects of the EPOC pathway, which also represents an institutional cultural change in how patients are viewed and managed perioperatively. NOPC patients met the same inclusion criteria as the EPOC patients and therefore represent a variety of both medical and surgical complexities.

Another limitation is the inability to know the extent of compliance with the full EPOC pathway for individual patients. There was some expected variation in compliance for each of the individual directives within EPOC, but the goal of this study was to assess overall cost changes of a multimodal EPOC pathway, even when some subtle variations might occur.

\section{Conclusions}

In a single institution, implementation of a multimodal EPOC pathway led to significant and quantifiable improvements in target metrics for adult patients undergoing major spine surgery. LOS was significantly lowered at both the intensive care and overall hospital level. The implementation of an EPOC pathway also achieved direct, indirect, and total cost savings in elective major spine surgery.

\section{Acknowledgments}

We thank Denise Leverentz, MBA, for her assistance in data collection.

\section{References}

1. Ali ZS, Ma TS, Ozturk AK, Malhotra NR, Schuster JM, Marcotte PJ, et al: Pre-optimization of spinal surgery patients: Development of a neurosurgical enhanced recovery after surgery (ERAS) protocol. Clin Neurol Neurosurg 164:142-153, 2018

2. Bacchin MR, Ceria CM, Giannone S, Ghisi D, Stagni G, Greggi T, et al: Goal-directed fluid therapy based on stroke volume variation in patients undergoing major spine surgery in the prone position: a cohort study. Spine (Phila Pa 1976) 41:E1131-E1137, 2016

3. Cerantola Y, Valerio M, Persson B, Jichlinski P, Ljungqvist $\mathrm{O}$, Hubner M, et al: Guidelines for perioperative care after radical cystectomy for bladder cancer: Enhanced Recovery After Surgery $\left(\right.$ ERAS $\left.^{\circledR}\right)$ society recommendations. Clin Nutr 32:879-887, 2013

4. Dagal A, Bellabarba C, Bransford R, Zhang F, Chesnut RM, O'Keefe GE, et al: Enhanced perioperative care for major spine surgery. Spine (Phila Pa 1976) [epub ahead of print]. 2018

5. Dort JC, Farwell DG, Findlay M, Huber GF, Kerr P, SheaBudgell MA, et al: Optimal perioperative care in major head and neck cancer surgery with free flap reconstruction: a consensus review and recommendations from the Enhanced Recovery After Surgery Society. JAMA Otolaryngol Head Neck Surg 143:292-303, 2017 
6. Feldheiser A, Aziz O, Baldini G, Cox BPBW, Fearon KCH, Feldman LS, et al: Enhanced recovery after surgery (ERAS) for gastrointestinal surgery, part 2: consensus statement for anaesthesia practice. Acta Anaesthesiol Scand 60:289-334, 2016

7. Fletcher ND, Andras LM, Lazarus DE, Owen RJ, Geddes BJ, Cao J, et al: Use of a novel pathway for early discharge was associated with a $48 \%$ shorter length of stay after posterior spinal fusion for adolescent idiopathic scoliosis. J Pediatr Orthop 37:92-97, 2017

8. Grasu RM, Cata JP, Dang AQ, Tatsui CE, Rhines LD, Hagan $\mathrm{KB}$, et al: Implementation of an Enhanced Recovery After Spine Surgery program at a large cancer center: a preliminary analysis. J Neurosurg Spine 29:588-598, 2018

9. Hansen RN, Pham AT, Böing EA, Lovelace B, Wan GJ, Miller TE: Comparative analysis of length of stay, hospitalization costs, opioid use, and discharge status among spine surgery patients with postoperative pain management including intravenous versus oral acetaminophen. Curr Med Res Opin 33:943-948, 2017

10. Kehlet H: Multimodal approach to control postoperative pathophysiology and rehabilitation. Br J Anaesth 78:606617, 1997

11. Lassen K, Coolsen MME, Slim K, Carli F, de Aguilar-Nascimento JE, Schäfer M, et al: Guidelines for perioperative care for pancreaticoduodenectomy: Enhanced Recovery After Surgery $\left(\right.$ ERAS $\left.^{\circledR}\right)$ Society recommendations. World J Surg 37:240-258, 2013

12. Loftus RW, Yeager MP, Clark JA, Brown JR, Abdu WA, Sengupta DK, et al: Intraoperative ketamine reduces perioperative opiate consumption in opiate-dependent patients with chronic back pain undergoing back surgery. Anesthesiology 113:639-646, 2010

13. Nelson G, Altman AD, Nick A, Meyer LA, Ramirez PT, Achtari C, et al: Guidelines for pre- and intra-operative care in gynecologic/oncology surgery: Enhanced Recovery After Surgery $\left(\right.$ ERAS $\left.^{\circledR}\right)$ Society recommendations-Part I. Gynecol Oncol 140:313-322, 2016

14. Nielsen RV, Fomsgaard JS, Siegel H, Martusevicius R, Nikolajsen L, Dahl JB, et al: Intraoperative ketamine reduces immediate postoperative opioid consumption after spinal fusion surgery in chronic pain patients with opioid dependency: a randomized, blinded trial. Pain 158:463-470, 2017

15. Papanastassiou I, Anderson R, Barber N, Conover C, Castellvi AE: Effects of preoperative education on spinal surgery patients. SAS J 5:120-124, 2011

16. Raman T, Nayar SK, Liu S, Skolasky RL, Kebaish KM: Costeffectiveness of primary and revision surgery for adult spinal deformity. Spine (Phila Pa 1976) 43:791-797, 2018

17. Raudenbush BL, Gurd DP, Goodwin RC, Kuivila TE, Ballock RT: Cost analysis of adolescent idiopathic scoliosis surgery: early discharge decreases hospital costs much less than intraoperative variables under the control of the surgeon. J Spine Surg 3:50-57, 2017

18. Sanders AE, Andras LM, Sousa T, Kissinger C, Cucchiaro $\mathrm{G}$, Skaggs DL: Accelerated discharge protocol for posterior spinal fusion patients with adolescent idiopathic scoliosis decreases hospital postoperative charges 22. Spine (Phila Pa 1976) 42:92-97, 2017

19. Sing DC, Khanna R, Shaw JD, Metz LN, Burch S, Berven $\mathrm{SH}$ : Increasing rates of surgical management of multilevel spinal curvature in elderly patients. Spine Deform 4:365372,2016

20. Soroceanu A, Ching A, Abdu W, McGuire K: Relationship between preoperative expectations, satisfaction, and functional outcomes in patients undergoing lumbar and cervical spine surgery: a multicenter study. Spine (Phila Pa 1976) 37:E103-E108, 2012

21. Stowers MDJ, Lemanu DP, Hill AG: Health economics in Enhanced Recovery After Surgery programs. Can J Anaesth 62:219-230, 2015

22. Temple-Oberle C, Shea-Budgell MA, Tan M, Semple JL, Schrag C, Barreto M, et al: Consensus review of optimal perioperative care in breast reconstruction: Enhanced Recovery after Surgery (ERAS) Society recommendations. Plast Reconstr Surg 139:1056e-1071e, 2017

23. Thorell A, MacCormick AD, Awad S, Reynolds N, Roulin D, Demartines N, et al: Guidelines for perioperative care in bariatric surgery: Enhanced Recovery After Surgery (ERAS) Society recommendations. World J Surg 40:2065-2083, 2016

24. Wainwright TW, Immins T, Middleton RG: Enhanced recovery after surgery (ERAS) and its applicability for major spine surgery. Best Pract Res Clin Anaesthesiol 30:91-102, 2016

25. Wang MY, Chang PY, Grossman J: Development of an enhanced recovery after surgery (ERAS) approach for lumbar spinal fusion. J Neurosurg Spine 26:411-418, 2017

\section{Disclosures}

The authors report no conflict of interest concerning the materials or methods used in this study or the findings specified in this paper.

\section{Author Contributions}

Conception and design: Dagal, Carr, Zhang, Bransford, Bellabarba. Acquisition of data: Dagal, Carr. Analysis and interpretation of data: Dagal, Carr, Saigal. Drafting the article: Dagal, Carr. Critically revising the article: Dagal, Carr, Saigal. Reviewed submitted version of manuscript: all authors. Approved the final version of the manuscript on behalf of all authors: Dagal. Statistical analysis: Dagal, Carr. Administrative/technical/material support: Dagal, Carr. Study supervision: Dagal, Carr.

\section{Correspondence}

Armagan Dagal: Harborview Medical Center, University of Washington, Seattle, WA.dagal@uw.edu. 